\title{
Filaire cardiaque chez un Manchot des Galapagos Spbeniscus mendiculus
}

\author{
Par Alain-G. CHABAUD et Gordon H. BALL
}

Grâce à l'obligeance du Dr. Hubert C. Johnstone, Pathologiste vétérinaire au Livestock Department, County of San Diego, nous avons reçu deux mâles et trois femelles d'une Filaire récoltée dans le cœur d'un Manchot des îles Galapagos, Spheniscus mendiculus Sund, qui était mort en captivité au Parc Zoologique de San Diego. Les spécimens fixés dans l'alcool-formol-glycérine n'ont pu être disséqués facilement et il serait bon que notre description soit vérifiée, particulièrement en ce qui concerne les papilles cloacales et le spicule gauche.

Le Dr. Johnstone, à qui nous adressons nos vifs remerciements, indique que, bien que les Vers aient été trouvés dans le cœur: «The lung pathology and the pathology of the air sac immediately over the lung was quite severe. ».

\section{Description :}

Corps cylindrique, relativement trapu pour une Filaire, recouvert d'une cuticule épaisse à stries transversales peu marquées.

Extrémité céphalique tronquée. Bouche circulaire très petite. Huit papilles céphaliques et deux amphides en situation normale, figurées en C. La cuticule dessine autour de la bouche un rectangle mal limité à peine saillant, et l'on ne peut donc parler d'un plateau céphalique. L'œsophage comprend une partie musculaire remarquablement courte, où la lumière de l'organe est étroite et rectiligne ; la partie glandulaire est longue et a un aspect très anormal, d'autant plus que la lumière de l'organe est large et finement plissée ; un éclaircissement très puissant des spécimens par le chloral-lactophénol a été nécessaire pour déceler l'union œsophago-intestinale. L'anneau nerveux entoure la partie antérieure de l'œsophage glandulaire. Le pore excréteur et une paire de très petites diérides sont situés un peu en arrière de l'anneau nerveux. Il n'y a pas d'ailes latérales.

Male : Corps long de $24 \mathrm{~mm}$, large de $290 \mu$. Esophages musculaire et glandulaire, longs respectivement de $115 \mu$ et $990 \mu$. Anneau nerveux à $195 \mu$ et pore excréteur à $230 \mu$ de l'apex. Les quatre millimètres postérieurs du corps sont enroulés en formant environ deux tours de spire; tout le long de cette zone, la cuticule ventrale 

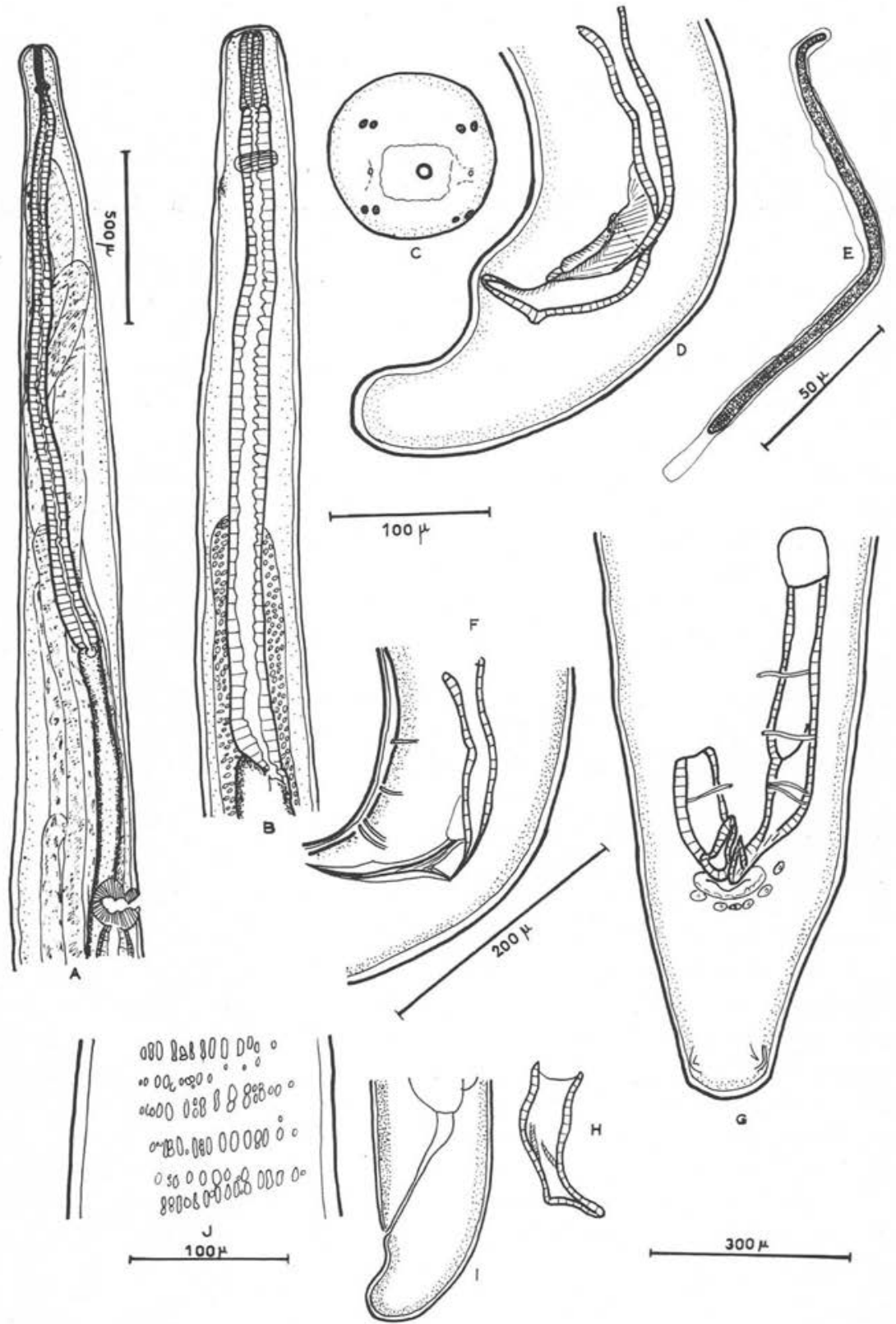

Paronchocerca straeleni. - A : Extrémité antérieure de la femelle, vue latérale. B : id. mâle. C : Tête de la femelle, vue apicale. D : Extrémité postérieure du mâle, vue latérale. E : Microfilaire. F : Spicule gauche. G : Extrémité postérieure du mâle, vue ventrale. H: Spicule droit. I: Queue de la femelle, vue latérale. J. : Ornementation cuticulaire de la queue du mâle. A : $500 \mu$. B, I: $300 \mu$. C, D, G, J : $100 \mu$. E. : $50 \mu$. F, H : $200 \mu$ 
porte une ornementation en bandes transversales un peu irrégulières, chaque bande étant constituée d'une quinzaine d'éléments bacilliformes saillants (fig. J).

Queue longue de $120 \mu$, arrondie à l'extrémité, ne portant que des papilles presque complètement atrophiées. En dehors des phasmides subterminales, nous avons cru voir cinq ou six papilles formant un arc de cercle sur la marge postérieure du cloaque et cinq papilles (une à droite et quatre à gauche) précloacales. Ces dernières n'ont pas été retrouvées sur le second spécimen disponible et ne sont peut-être que des sillons cuticulaires sans signification.

Spicules inégaux. Le droit long de 110 ou $130 \mu$ est trapu, en forme de pied et le manche est peu distinct de la lame. Le gauche, long de $260 \mu$ environ, est constitué par un manche long de $160 \mu$ et une lame qui paraît ressembler à celle d'une Setaria, mais ne pourrait être décrite correctement qu'après avoir été isolée par dissection. Gubernaculum absent.

Femelle : Corps long de $38 \mathrm{~mm}$ et large de $430 \mu$. Esophage total long de $1,85 \mathrm{~mm}$, la partie musculaire étant de $170 \mu$. Anneau nerveux à $220 \mu$ de l'apex. Diérides et pore excréteur au même niveau, à $110 \mu$ en arrière de l'anneau nerveux. Vulve à 2,65 mm de l'extrémité antérieure. L'ovéjecteur comprend un sphincter prévulvaire dont la lumière est tordue en spirale, puis un tube musculaire impair long de $1,5 \mathrm{~mm}$ dirigé vers l'arrière. Ce tube se courbe vers l'avant un peu avant sa division en deux branches. Les deux utérus, pleins de microfilaires, montent dans la région œsophagienne puis suivent un trajet récurrent opistodelphe jusqu'à l'extrémité postérieure où se trouvent les oviductes et ovaires. Queue courte $(140 \mu)$ et arrondie. Les microfilaires extraites des utérus et examinées dans le lactophénol sont pourvues d'une gaine. Elles sont longues de $155 \mu$ et larges de $4 \mu$.

La seconde femelle a un œsophage long de $1,5 \mathrm{~mm}$ et la vulve s'ouvre à $3,3 \mathrm{~mm}$ de l'apex. La vulve est donc ici beaucoup plus éloignée de l'extrémité postérieure de l'œsophage.

Le matériel type est déposé au Muséum National d'Histoire Naturelle (Bocal N 147, $\left.n^{\circ} 360 \mathrm{G}\right)$.

\section{Discussion :}

L'espèce appartient au genre Paronchocerca Peters 1936 dont les limites avec le genre Aproctoides Chandler 1929 sont imprécises.

Le genre Aproctoides est basé sur la description d'un mâle ; la femelle est inconnue et cela entraîne de grandes difficultés dans la nomenclature. En effet, Ali (1956) décrit dans le genre une seconde espèce Aproctoides papillatus dont les femelles ont des microfilaires. Chabaud et Anderson (1959) placent donc le genre dans la famille des Onchocercidae et non dans celle des Filariidae, puisque cette famille est caractérisée par des femelles n'ayant pas de microfilaires.

Chabaud et Anderson ajoutent au genre une troisième espèce A. helicinum (Molin), redécrite par Wehr et Hwang (1957) et ayant également des larves de type microfilaire ; ils proposent la mise en synonymie avec Aproctoides du nom de genre Wymania créé par Wehr et Hwang pour la Filaria helicina de Molin. 
En $1961 a$, Sonin ajoute au genre une nouvelle espèce Aproctoides striata qui, cette fois, a des femelles ovipares et n'appartient donc pas à la famille des Onchocercidae, mais à celle des Filariidae.

Il apparaît qu'Aproctoides striata Sonin est plus proche de l'espèce type, Aproctoides lissum, que ne l'est Aproctoides papillatus Ali. L'aspect des spicules en particulier semble pouvoir permettre le rapprochement. Sonin $(1961 \mathrm{~b})$ paraît donc tout à fait justifié en replaçant le genre Aproctoides Chandler dans les Filariidae Aproctinae avec $A$. lissum comme espèce type et $A$. striata Sonin comme seconde espèce.

Les deux autres espèces $A$. helicinum et $A$. papillatus ayant des microfilaires restent dans les Onchocercidae-Splendidofilariinae, mais il est difficile d'accepter le nouveau genre Pseudaproctoides Sonin 1961 avec $P$. papillatus (Ali) comme espèce type. Il faut, ou bien les incorporer au genre Paronchocerca, ou bien les classer dans le genre Wymania qui a priorité.

La première solution nous semble préférable car, chez les Filaires, l'ornementation cuticulaire est un mauvais élément de diagnose générique. Nous proposons donc la mise en synonymie des genres Wymania Wehr et Hwang 1957 et Pseudaproctoides Sonin 1961 avec le genre Paronchocerca Peters 1936 (1).

Celui-ci comprend donc 8 espèces :

P. sanguinis-ardeae (Léger et Noc 1921) sp. inquirenda, parasite dans le cœur d'un Héron à Dakar, n'a aucune raison particulière pour être assimilée à notre espèce.

P. bambusicolae (H. C. Li 1933), parasite des tissus sous-cutanés d'un Galliforme en Chine, a des annelures sur la cuticule et une vulve pré-œsophagienne.

$P$. ciconiarum Peters 1936, parasite du cœur d'Ardeiformes en Afrique, diffère de notre matériel par les annelures de la cuticule et la plus grande taille des spicules.

P. tonkinensis (Chow 1939) parasite du cœur (?) d'Ardéiformes au Viet-Nam, a de annelures sur la cuticule et un œsophage musculaire plus développé que chez notre espèce.

P. rousseloti Chabaud et Biocca 1951, parasite du cœur de Galliformes africains, a des spicules de grande taille. La bande ornée du mâle a des lignes transversales avec 7 à 12 éléments (une quinzaine chez le parasite du Manchot).

P. mirzai Ali 1956, parasite du cœur d'un Galliforme indien aurait un œsophage très court. La cuticule a des rugosités annulaires.

$P$. papillatus (Ali 1956) nov. comb., des poumons d'un Galliforme indien, a une vulve préœsophagienne et des spicules plus grands.

P. helicina (Molin 1858) nov. comb., du cerveau des Anhingas, a une vulve très antérieure par rapport à la longueur de l'œsophage.

(1) Il est parfois très difficile de voir l'union entre l'œsophage glandulaire et l'intestin. Il serait utile de vérifier Paraprocta brevicauda (Chandler 1924), car si l'espèce avait un œesophage glandulaire faisant suite à l'œsophage musculaire rien ne l'opposerait plus au genre Paronchocerca et dans ce cas Paroncherca Peters 1936 tomberait en synonymie de Paraprocta Maplestone 1931. 
L'espèce se distingue donc aisément des formes décrites précédemment et nous proposons pour elle le nom de Paronchocerca straeleni n. sp. pour la dédier à la mémoire du Professeur Victor van Straelen qui fut Président de la fondation Charles Darwin pour les Galapagos.

Cette Filaire est, à notre connaissance, la première qui soit décrite chez un Oiseau de la Sous-classe des Impennes. Elle se distingue surtout par la brièveté de l'œsophage musculaire.

On peut noter également une inégalité particulièrement marquée entre les deux spicules. Nous ne sommes pas sûrs cependant que Sonin $(1961 b)$ ait raison de placer le genre dans les Onchocercinae, sous-famille qui, dans la classification de Chabaud et Anderson, ne compte aucun parasite d'Oiseaux. L'égaiıté entre les deux spicules ne nous apparaît pas comme un très bon caractère phylétique; il semble que les Filaires à spicules égaux dérivent de formes à spicules inégaux. Paronchocerca semble un genre archaïque ayant peut-être certaines affinités avec des parasites de Reptiles tels que Oswaldofilaria ou Macdonaldius, mais l'atrophie des papilles cloacales et leur concentration autour du cloaque s'observent surtout chez de nombreuses Splendidofilariinae (sensu Anderson 1961). C'est pourquoi nous sommes d'avis de placer ce genre à la fois archaïque et synthétique à la base de la sous-famille des Splendidofilariinae.

\section{Résumé}

Description de Paronchocerca straeleni n. sp., Filaire parasite dans le cœur du Manchot des Galapagos: Spheniscus mendiculus Sund.

Sonin ayant replacé l'espèce type du genre Aproctoides dans les Aproctinae (Filaires ovipares), nous pensons que les deux espèces, ayant des Microfilaires, qui étaient classées dans Aproctoides, peuvent entrer dans le genre Paronchocerca.

Nous proposons donc de considérer les genres Wymania Wehr et Hwang 1957 et Pseudaproctoides Sonin 1961 comme synonymes de Paronchocerca.

Nous exposons brièvement pourquoi nous classons ce genre, qui paraît avoir des caractères archaïques et synthétiques, à la base de la sous-famille des Splendidofilariinae (sensu Anderson 1961).

\section{Bibliographie}

Ali (Syed Mehdi), 1956. - Studies on the nematode parasites of fishes and birds found in Hyderabad state. Indian J. Helminth., 8, 1-83.

ANDERSON (Roy C.), 1961. - Splendidofilaria wehri n. sp. with a revision of Splendidofilaria and related genera. Can. J. Zool., 39, 201-207.

Chabaud (A.-G.) \& Anderson (R.-C.), 1959. - Nouvel essai de classification des Filaires (superfamille des Filarioidea) II... Ann. Parasitol., 34, 64-87.

Annales de Parasitologie humaine et comparée (Paris), t. 39, 1964, $\mathrm{n}^{\circ} 5$ 
Chabaud (A.-G.) \& Biocca (Ettore), 1951. - Description d'une nouvelle filaire cardiaque et remarques sur le genre Paronchocerca Peters 1936. Ann. Parasitol., 26, 338-345.

Chander (A. C.), 1924. - New Filaria from Indian Birds. Parasitol., 16, 398-404.

ChANDler (A.C.), 1929. - Some new genera and species of nematode worms, Filarioidea from animals dying in the Calcutta zoological garden. Proc. U.S. Nat. Mus. (2777), 75 , art. $6,1-10$, pls. 1-3.

CHow (C. Y.), 1939. - Notes sur quelques Nématodes de l'Indochine française. Ann. Parasitol., 17, 21-31.

LI (H. C.), 1933. - Report on a collection of parasitic nematodes, mainly from North China. Part. I. Filarioidea. Parasitol., 25, 192-223.

Maplestone (P. A.), 1931. - Parasitic Nematodes obtained from animals dying in the Calcutta zoological Gardens. Rec. Indian Mus., 33, II, 71-171.

Noc (F.), 1921. - Filaire sanguicole du Héron Goliath du Sénégal. C.R. Soc. Biol., 84, 69-71.

Peters (B. G.), 1936. - Paronchocerca ciconiarum n. g. n. sp. from the Saddle billed Stork in West Africa. J. Helminth., 14, 1-10.

SonIN (M. D.), 1961 a. - Les filaires des Oiseaux du Turkménistan. Helminthol., 3, 322-339.

SonIN (M. D.), 1961 b. - Révision des Nématodes de la sous-famille Splendidofilariinae Chabaud et Choquet 1953. Trudi Gelm. Lab., 11, 242-250.

Wehr (E. E.) et Hwang (J. C.), 1958. - Systematic position of Filaria helicina Molin, 1858, from the brain cavity of the snakebird, Anhinga anhinga. J. Parasitol., 43, 649-655.

[Laboratoire de Zoologie (Vers), Museum National d'Histoire Naturelle, 57, rue Cuvier, Paris et Department of Zoology, University of California, Los Angeles, Californie (U.S.A.)]. 